\title{
Maternal heterozygous mutation in CHEK1 leads to mitotic arrest in human zygotes
}

\section{Dear Editor,}

The first mitotic division in zygotes is crucial for the beginning of the life cycle for the human. After fertilization, zygotes reactivate cell cycle, both paternal and maternal genomes replicate and reprogram to become totipotent. In the meantime, the male and female pronucleus move to the center of the zygote and merge. Then zygotes enter the metaphase, and sister chromatids separate into two daughter cells (Eckersley-Maslin et al., 2018; Reichmann et al., 2018). This is a sensitive time window and many perturbances may cause the first mitosis to fail.

We discovered a family that the female adults were infertile. One female patient (proband III-7) underwent in vitro fertilization (IVF) treatment. The proband had regular menstrual cycles and sex hormone levels. She had undergone only one IVF cycle with classical controlled ovarian hyperstimulation protocol. Seventeen oocytes were retrieved and fertilized in vitro. Eight out of 15 metaphase II (MII) oocytes presented two obvious pronuclei during the fertilization checking. However, all embryos failed to complete the first cleavage (Table 1). Further investigation found that one sister (III-4) had undergone three failed IVF attempts for the same reason, two other sisters (III-3 and III-6) were infertile for unknown causes, and two sisters (III-2 and III-5) had healthy children. With the patients' consent, whole-exome sequencing was carried out in all of the infertile subjects. After the pedigree analysis, we found that a heterozygous missense variant in the checkpoint kinase 1 gene (CHEK1), c.1325G >A (p. Arg442GIn), namely R442Q, was shared by all the infertile sisters (Fig. 1A). The variant was confirmed by Sanger sequencing in the whole family members (Fig. 1B). The results suggested that each infertile subject inherited the variant from their father II-8, and the variant was segregated within the whole family (Fig. 1A and 1B). CHEK1 protein plays an essential role in the DNA damage response (Sidi et al., 2008; Reinhardt and Yaffe, 2009) and is conserved across species (Fig. S1A). Interestingly, although the same CHEK1 $R 442 Q$ variant is expressed in somatic cells, based on RT-PCR and Sanger sequencing analysis of the peripheral blood samples from III-2, 3, 4, 5, 6 and 7 (Figs. 1C, S1B and S1C), heterozygous carriers are all healthy. We also analyzed the transcriptome profile of human oocytes, 1 and 2-cell embryos, CHEK 1 transcripts are present at high levels in germinal vesicle (GV) and MII oocytes, zygotes, and 2-cell embryos (Fig. 1D). Therefore, it should exist as a maternal protein.

To test how CHEK1 R442Q protein affects zygote division, we cloned mouse Chk1, whose amino acid sequence is 93.07\% identical to that of the human CHEK1, and generated the same Chk1 R442Q mutation as found in the infertile patient. Upon overexpression of wild type (WT) or R442Q Chk1 protein in mouse zygotes by mRNA injection, we found that more than $60 \%$ of the Chk1 R442Q zygotes failed to divide into 2-cell embryos, while overexpression of Chk1 WT did not affect the first mitosis (Fig. 1E-G). It has been shown that overexpression of Chk1 in mouse GV oocytes caused meiosis I arrest (Chen et al., 2012). Interestingly, when we overexpressed Chk1 WT and R442Q in GV oocytes, the $\mathrm{R} 442 \mathrm{Q}$ caused significantly more oocyte arrest in metaphase I than WT (Fig. S2C-E). We analyzed Chk1 protein localization based on the immunofluorescence signal, the Chk1 WT and R442Q proteins localized both in nucleus and cytoplasm, with a stronger localization at nucleus and on the spindle during mitosis and meiosis (Fig. S2A and S2F). The amount of overexpressed mutant protein is less than the overexpressed WT protein, based on their respective mean fluorescence intensity (MFI) (Fig. S2B and S2G), suggesting that the $R 442 Q$ change did not stabilize Chk1 protein.

Table 1. Oocyte and embryo phenotypes of IVF attempt for proband III-7 female patient carrying the CHEK1 C.1325G>A (R442Q) variant.

\begin{tabular}{lllllllll}
\hline Individual & $\begin{array}{l}\text { Age } \\
\text { (years) }\end{array}$ & $\begin{array}{l}\text { Duration of } \\
\text { infertility } \\
\text { (Years) }\end{array}$ & $\begin{array}{l}\text { IVF and } \\
\text { ICSI } \\
\text { attempts }\end{array}$ & $\begin{array}{l}\text { Retrieved } \\
\text { oocytes }\end{array}$ & $\begin{array}{l}\text { MII } \\
\text { oocytes }\end{array}$ & $\begin{array}{l}\text { OPN } \\
\text { oocytes }\end{array}$ & $\begin{array}{l}\text { 2PN } \\
\text { oocytes }\end{array}$ & $\begin{array}{l}\text { Cleaved } \\
\text { fertilized } \\
\text { oocytes }\end{array}$ \\
\hline III-7 & 29 & 2 & First IVF & 17 & 15 & 7 & 8 & 0 \\
\hline
\end{tabular}


A

Family 1

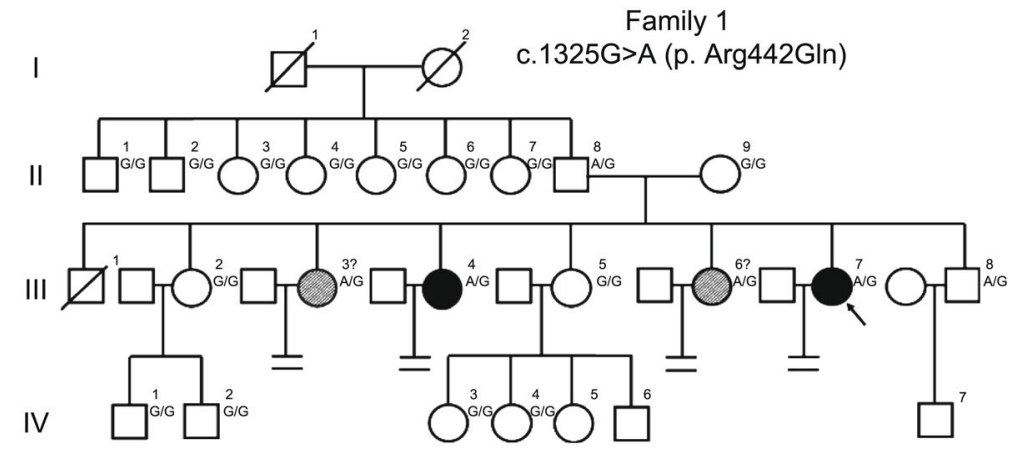

B

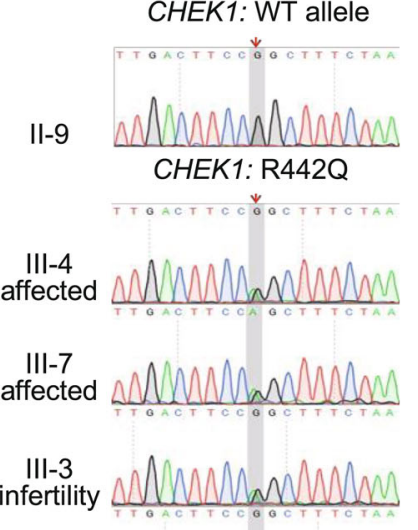

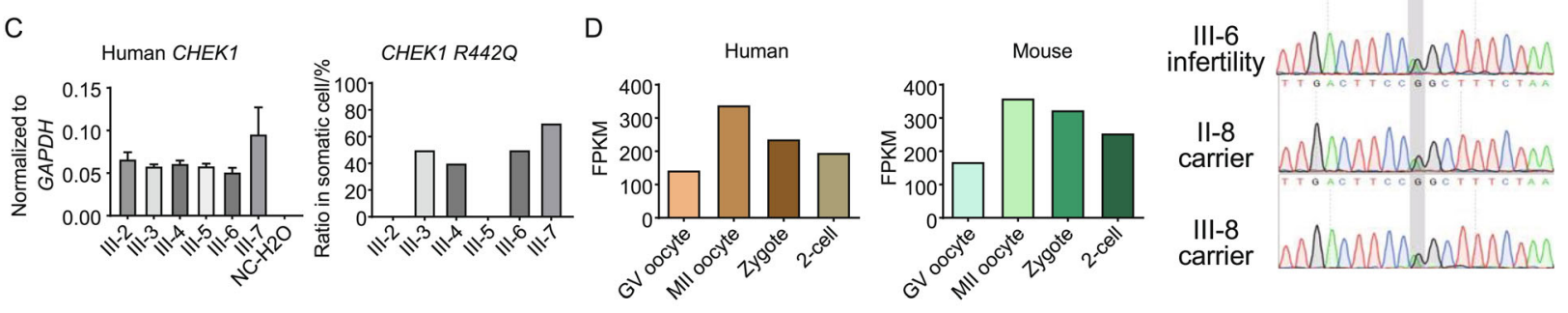

E

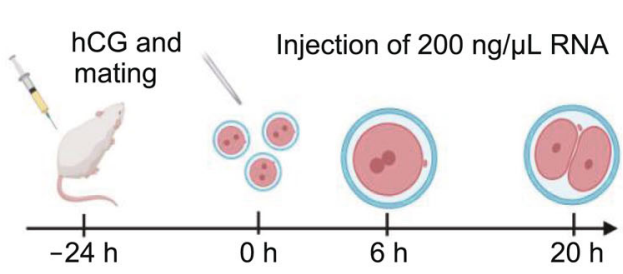

$\mathrm{F}$

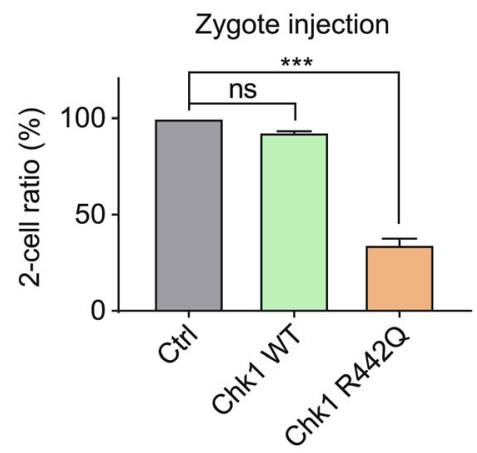

$\mathrm{H}$

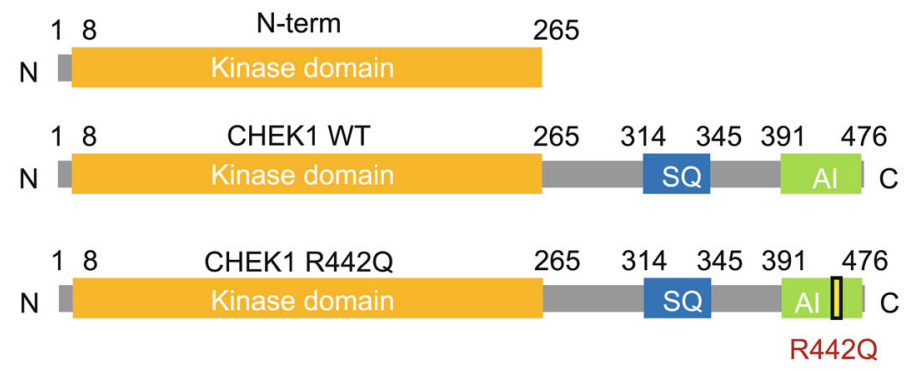

G
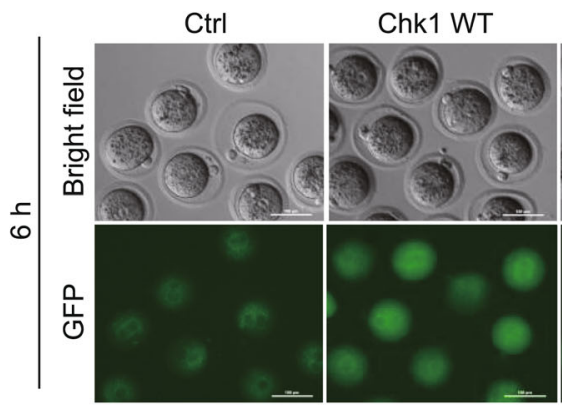

Chk1 R442Q
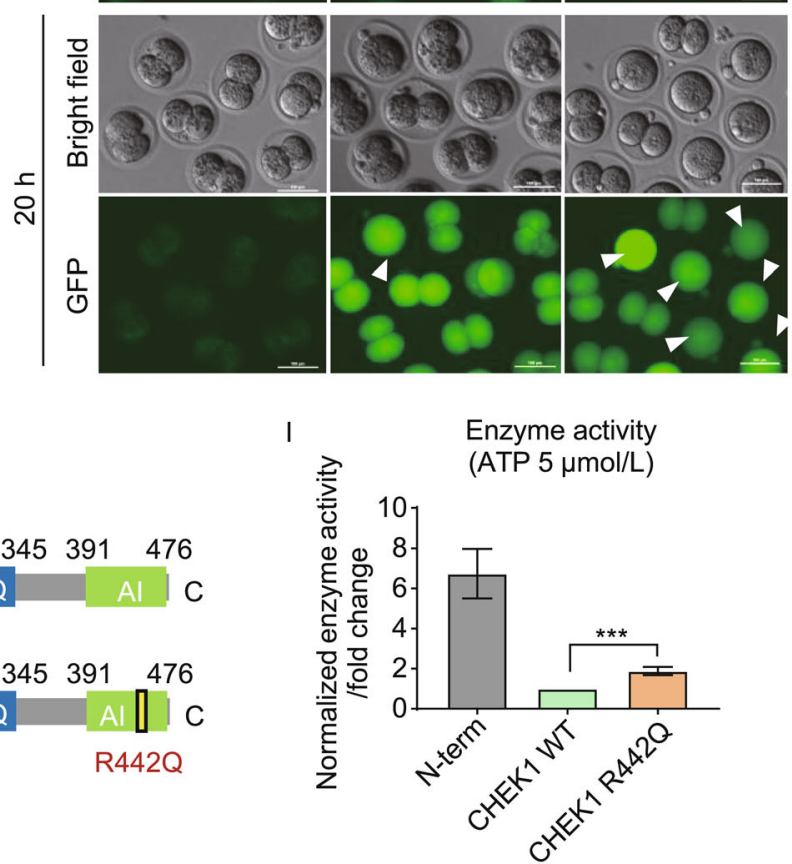
4 Figure 1. First mitosis failure in zygotes from CHEK1 c.1325G >A (p. Arg442GIn) females. (A) Genetic analysis in the families carrying CHEK1 c.1325G>A (p. Arg442GIn). Black circles indicate the affected individuals, and the circles with slashes indicate the suspected individuals (III-4 and III-7 had been confirmed during IVF cycles, III-3 and III-6 females were infertile, possibly caused by the arrest of the embryo cleavage). The black arrowhead indicates the proband. (B) Sanger sequencing result of $C H E K 1$ c.1325G>A (R442Q) mutation in affected, infertile, and carrier individuals. Double peak at the 1,325 position highlights the mutated nucleotide. (C) QPCR result confirmed that $C H E K 1 \mathrm{R} 442 \mathrm{Q}$ transcripts are present in patients' somatic cells. Left panel, the expression levels of CHEK1 mRNA in human blood cells. Right panel, the ratio of CHEK1 R442Q mutant transcript in patient somatic cells verified by Sanger sequencing. (D) CHEK1 expression levels in human and mouse GV oocyte to 2-cell embryo based on RNA-Sequencing data from GSE101571, GSE36552, and GSE71434. (E) Schematic of overexpressing Chk1 WT and $\mathrm{R} 442 \mathrm{Q}$ in mouse zygotes by mRNA microinjection (Created with BioRender.com). (F) Bar plot showing Chk1 R442Q arrest the first cleavage. Ctrl, $n=23$. WT, $n=48$. R442Q, $n=51$. All pooled from 2 separate experiments; one-way analysis of variance (ANOVA), Bonferroni's test for individual comparisons. Bars are means \pm s.e.m. (G) Representative live cell images of zygotes microinjected with mRNA encoding Chk1 WT and R442Q GFP fusion protein. White arrowheads point to the arrested embryos. Time is as indicated. Scale bar, $100 \mu \mathrm{m}$. (H) Schematic of human CHEK1 protein domains. (I) CHEK1 $\mathrm{R} 442 \mathrm{Q}$ protein showed higher kinase enzyme activity compared to WT. Luciferase based kinase assay normalized to WT group. 9 tests from 3 separate experiments. Two-sided Student's $t$-test. Columns are means \pm s.e.m.

As the activation of CHEK1 kinase in response to DNA damage will cause cell cycle arrest in the $\mathrm{G}_{2}$ phase (Reinhardt and Yaffe, 2009), we hypothesize that the R442Q mutation may cause an increase in CHEK1 protein kinase activity. Therefore, we generated the $\mathrm{N}$-terminus kinase domain, the full-length, and the full-length R442Q mutant CHEK1 proteins and performed the kinase assay (Fig. 1H). Indeed, the $\mathrm{N}$-terminus kinase domain had very high kinase activity, as reported by several studies (Goto et al., 2015; Han et al., 2016; Emptage et al., 2017). The R442Q mutant protein had about twice as high kinase activity as the WT protein (Figs. 1I, S1D and S1E).

There have been many studies about CHEK1 and checkpoint kinase 2 (CHEK2) function in the context of human cancers. We looked for identified disease-related variants within CHEK1 and CHEK2 genes in the Human gene mutation database (HGMD). There are only 4 entries for CHEK1 but 345 entries for CHEK2 (Table S1). Four variants in CHEK1 are all within the sequence encoding the kinase domain and associated with cancers. A high
Figure 2. A graded Chk1 activity differentially modulates DNA damage response and cell cycle progression in the zygote. (A) Representative $\mathrm{YH} 2 \mathrm{AX}$ immunostaining images of Ctrl, Chk1 WT, and R442Q zygotes. Yellow circles mark the paternal and maternal pronucleus. PB, polar body. Cyan, DAPI; pink, yH2AX; gray, Chk1. Scale bar, $20 \mu \mathrm{m}$. (B) Dot plot quantification of $\mathrm{YH} 2 \mathrm{AX}$ signal in arrested $\mathrm{R} 442 \mathrm{Q}$ mouse zygotes compared to control non-injected (Ctrl) and WT injected zygotes. Ctrl, $n=66$, WT, $n=28$. R442Q, $n=58$. All pooled from 2 separate experiments. One-way analysis of variance (ANOVA), Bonferroni's test for individual comparisons. Bars are means \pm s.e.m. (C) $\mathrm{yH} 2 \mathrm{AX}$ staining in CHEK1 inhibitor $(0.1 \mu \mathrm{mol} / \mathrm{L})$ rescued 2-cell embryos. Cyan, DAPI; pink, $\mathrm{YH} 2 \mathrm{AX}$; gray, Chk1. Scale bar, $20 \mu \mathrm{m}$. (D) Quantification of $\mathrm{YH} 2 \mathrm{AX}$ signal in $0.1 \mu \mathrm{mol} / \mathrm{L}$ CHEK1 inhibitor-treated or non-treated WT and R442Q 2-cell embryos. Chk1 WT embryos with or without inhibitor treatment, $n=15$ and 21. Chk1 R442Q embryos with or without inhibitor treatment, $n=8$ and 20. One-way analysis of variance (ANOVA), Bonferroni's test for individual comparisons. Bars are means \pm s.e.m. (E) $30 \mathrm{nmol} / \mathrm{L}$ CHEK1 inhibitor treatment rescued the development of Chk1 R442Q zygotes. Chk1 WT embryos with or without $30 \mathrm{nmol} / \mathrm{L}$ inhibitor treatment, $n=98$ and 106. Chk1 $\mathrm{R} 442 \mathrm{Q}$ embryos with or without $30 \mathrm{nmol} / \mathrm{L}$ inhibitor treatment, $n=106$ and 101. All pooled from 4 separated experiments. One-way analysis of variance (ANOVA), Bonferroni's test for individual comparisons. Bars are means \pm s.e.m. $(F)$ Representative images of Chk1 WT and $\mathrm{R} 442 \mathrm{Q}$ injected embryos with or without $30 \mathrm{nmol} / \mathrm{L}$ inhibitor treatment at $48 \mathrm{~h}$ post HCG. Scale bar, $100 \mu \mathrm{m}$. (G) $30 \mathrm{nmol} / \mathrm{L}$ CHEK1 inhibitor-treated embryos can develop to the blastocyst stage. Chk1 WT, $n=48$. Chk1 $\mathrm{R} 442 \mathrm{Q}, n=46$. All pooled from 2 separate experiments. Two-way analysis of variance (ANOVA), Bonferroni's test for individual comparisons. Bars are means \pm s.e.m. (H) Representative images of 8-cell and blastocysts developed from zygotes injected with mRNA of Chk1 WT and $\mathrm{R} 442 \mathrm{Q}$ then treated with $30 \mathrm{nmol} / \mathrm{L}$ CHEK1 inhibitor. The stage is as indicated. Scale bar, $100 \mu \mathrm{m}$. (I) Immunostaining of Oct4 and Nanog in blastocysts developed from WT or $\mathrm{R} 442 \mathrm{Q}$ zygotes treated with $30 \mathrm{nmol} / \mathrm{L}$ CHEK1 inhibitor. The dashed line highlights the ICM region. Blue, DAPI; green, Oct4; pink, Nanog. Scale bar, $20 \mu \mathrm{m}$. ICM, inner cell mass. (J) Statistic analysis of total cell number in blastocysts derived from Chk1 WT and R442Q injected zygotes with or without inhibitor treatment. WT embryos with or without $30 \mathrm{nmol} / \mathrm{L}$ inhibitor treatment, $n=19$ and 15. R442Q embryos with or without $30 \mathrm{nmol} / \mathrm{L}$ inhibitor treatment, $n=17$ and 13. All pooled from 2 separate experiments. One-way analysis of variance (ANOVA), Bonferroni's test for individual comparisons. Bars are means \pm s.e.m. $(K)$ Statistic analysis of the ratio of Oct4, and Nanog positive cells, related to $(\mathrm{J})$. 

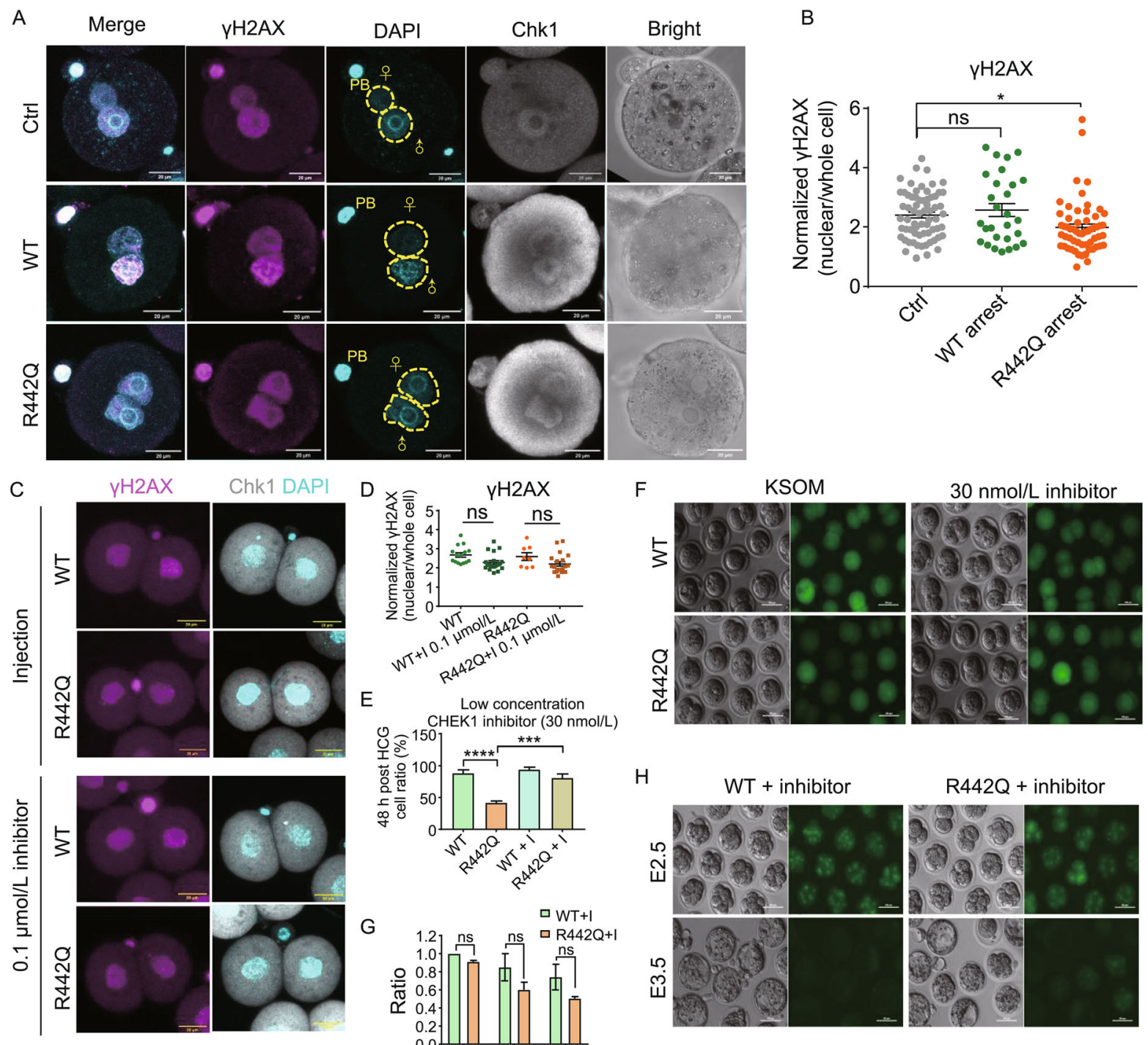

$\mathrm{H}$
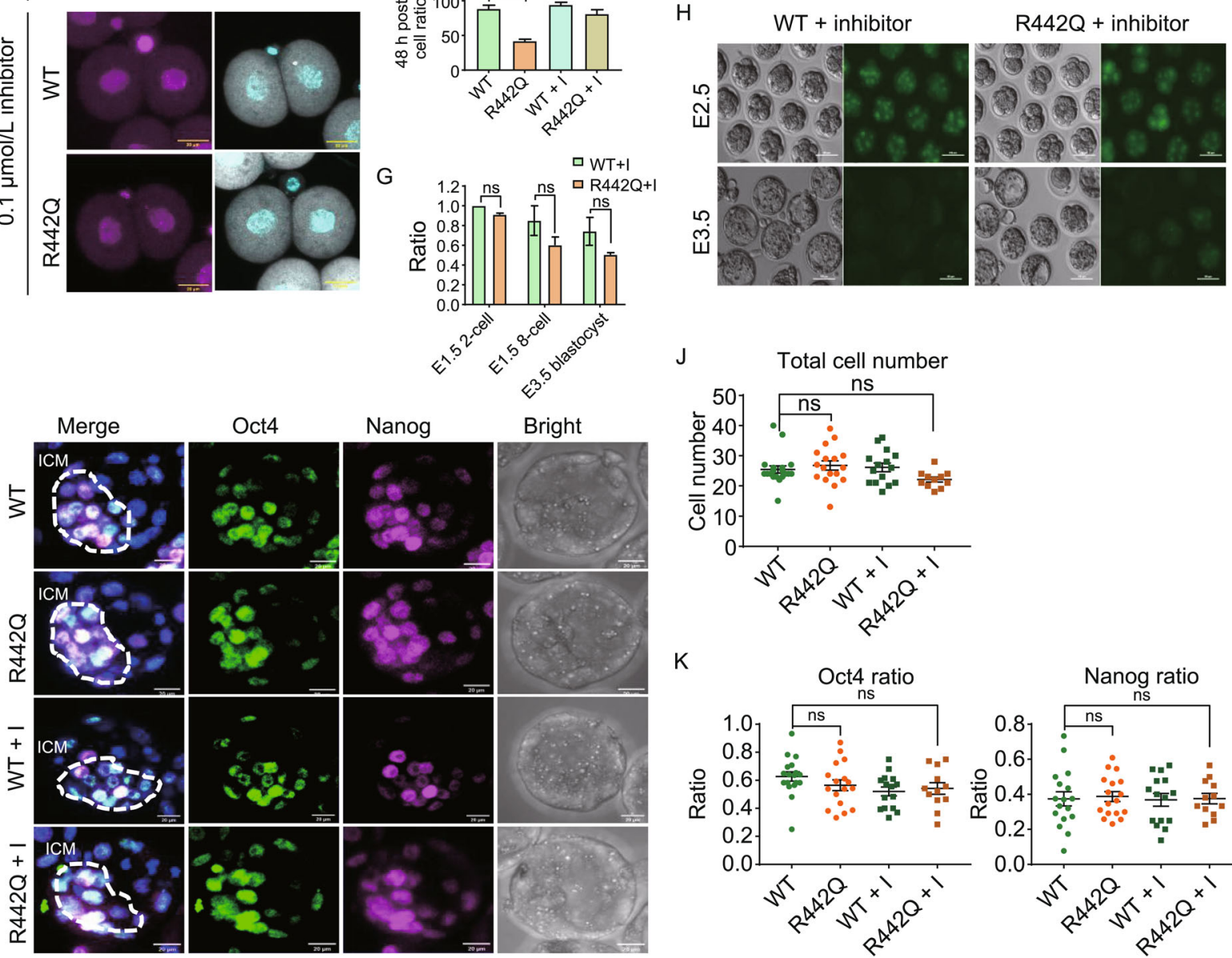

Bright

K

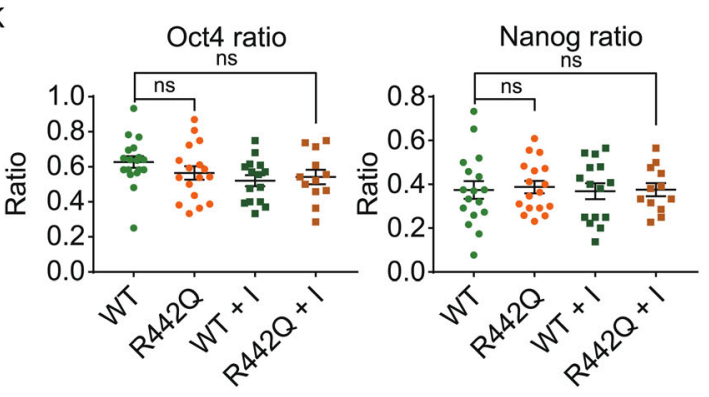


proportion of $\mathrm{CHEK} 2$ variants are related to breast cancers. There was no report about the CHEK1 c.G1325A, p.R442Q mutation. We speculate that this is likely due to female infertility caused by this mutation.

We next ask whether Chk1 R442Q may induce more DNA damage in zygotes. Interestingly, overexpression of Chk1 $\mathrm{R} 442 \mathrm{Q}$ reduced $\mathrm{YH} 2 \mathrm{AX}$ staining in the male pronucleus (Figs. 2A, 2B and S3A). Since R442Q mutant protein has higher kinase activity than the WT protein, we used a CHEK1 inhibitor CCT244747 to treat zygotes overexpressing WT or R442Q. As expected, CCT244747 rescued the first mitosis arrest caused by $R 442 Q$, nearly all treated R442Q zygotes divided into 2-cell embryos (Fig. S3B). However, regular CCT244747 dosage also increased DNA damage, as shown by stronger $\mathrm{YH} 2 \mathrm{AX}$ staining in treated WT or R442Q embryos (Fig. S3C). Since R442Q mutant has approximately double kinase activity than WT protein, we reasoned that reducing the kinase activity close to the normal level might be sufficient to overcome the first mitotic arrest. Indeed, $30 \mathrm{nmol} / \mathrm{L}$ of CCT244747 permitted R442Q zygotes to divide into 2-cell embryos and did not increase the $\mathrm{yH} 2 \mathrm{AX}$ signal (Figs. 2C, 2D and S3D-F). Moreover, $60 \%$ of $30 \mathrm{nmol} / \mathrm{L}$ CCT244747 treated R442Q zygotes developed to the blastocyst stage (Fig. 2E-H). We examined the expression of pluripotency genes and found that Oct4 and Nanog proteins are highly expressed in the inner cell mass of CCT244747 treated R442Q blastocysts (Fig. 2I). Treated WT and R442Q blastocysts also had a similar number of total cells and Oct4 and Nanog positive cells (Fig. 2J and $2 \mathrm{~K})$, suggesting that their development was not compromised.

We next performed transcriptome profiling of zygotes, 2-cell embryos, and blastocysts injected with mRNA encoding WT or R442Q with or without low dosage CHEK1 inhibitor treatment (Fig. S4A). Principal component analysis (PCA) and heatmap clustering showed that 1-cell arrested R442Q zygotes clustered together with PN5 WT zygotes (Fig. S4B and S4C), suggesting that the minor zygotic genome activation was not affected. At the late 2-cell stage, the transcriptome of $\mathrm{R} 442 \mathrm{Q}$ embryos, which managed to divide, clustered together with WT 2-cell embryos (Fig. S4B and $\mathrm{S} 4 \mathrm{C}$ ), indicating that the major zygotic genome activation occurred in these embryos. The CHEK1 inhibitor rescued WT and R442Q blastocysts clustered together, consistent with our previous observations (Figs. S4B, S4C and $2 \mathrm{I}-\mathrm{K})$. We analyzed differentially expressed transcripts in WT and R442Q zygotes, 2-cell embryos, and CHEK1 inhibitor rescued WT and R442Q blastocysts. The expression of zygotic genome activation genes and key developmental genes were largely unaffected (Fig. S4D and Table S2). Finally, we transferred CHEK1 inhibitor rescued $\mathrm{R} 442 \mathrm{Q} 2$-cell embryos into female mice and found that a similar number of pups were born compared with the WT embryo transferred group (Fig. S4E-G). These pups grew up to adulthood and had similar number of offspring as WT pups treated with or without the CHEK1 inhibitor (Fig. $\mathrm{S} 4 \mathrm{H}$ ). These results suggest that the low concentration of CHEK1 inhibitor treatment may relieve zygotes from the first mitotic division arrest caused by a slight tightening up of DNA damage checkpoint, and the rescued embryos would develop normally.

The discovery of the CHEK1 R442Q mutation and the first zygotic mitosis arrest phenotype in human reproduction has important implications. All the heterozygous female carriers are infertile, presumably due to failed mitosis in zygotes. As the zygotes contained mostly maternal proteins inherited from the egg, both CHEK1 WT and R442Q proteins should be present in the carrier's eggs. Based on the clinical observation during the IVF treatment, CHEK1 R442Q did not affect oocyte meiosis, suggesting that a small elevation in CHEK1 kinase activity does not block the first meiotic division in women. The II-8 and III-8 male carriers are healthy and do not have fertility problems, indicating that $\mathrm{CHEK} 1$ $\mathrm{R} 442 \mathrm{Q}$ does not affect sperm meiosis in men. CHEK1 is a ubiquitously expressed protein, and all the heterozygous individuals are healthy without cancer or autoimmune diseases based on informed consent health history surveys. Thus, CHEK1 R442Q should not affect most mitotic division of embryonic or adult cells. All the heterozygous females inherited the CHEK1 R442Q mutation from their father as their mother has two copies of the normal CHEK1 gene. Since the paternal genome starts to express after the major zygotic genome activation during the 4 to 8-cell stage in humans (Eckersley-Maslin et al., 2018), the CHEK1 R442Q protein derived from the mutated paternal copy of $\mathrm{CHEK} 1$ will likely be present from the 8-cell stage onwards. Therefore, the small elevation of CHEK1 kinase activity does not appear to arrest cell division after the 8-cell stage in human embryos. Thus, the CHEK1 R442Q protein seemed to affect the first mitotic division most seriously, indicating that the first mitosis is particularly sensitive to DNA damage checkpoint activation in humans. Moreover, the DNA damage checkpoint threshold may depend on the cell type, developmental stage, and species, based on the human phenotype and our results obtained in the mouse system. When we treat Chk1 $\mathrm{R} 442 \mathrm{Q}$ overexpressing mouse zygotes with a low dosage of CHEK1 inhibitor, they can escape the first mitotic arrest and develop normally. CHEK1 inhibitors are often used as cancer drugs. They inhibit the DNA damage checkpoint, making cancer cells accumulate DNA damage and die (Sidi et al., 2008; Reinhardt and Yaffe, 2009). Surprisingly, although a high dosage of CHEK1 inhibitor also induced DNA damage in zygotes, a low dosage of CHEK1 inhibitor rescued R442Q overexpression induced first mitosis arrest without eliciting any DNA damage judging by the $\mathrm{yH} 2 \mathrm{AX}$ staining, and the embryos developed normally. Chk1 R442Q overexpression also did not induce $\mathrm{yH} 2 \mathrm{AX}$ signal. The above results suggested that small up or down-regulation of CHEK1 kinase 
activity may not affect genome stability. Our findings also imply the possibility to use low dosage CHEK1 inhibitor treatment to release cells from the low-level genome stressinduced cell cycle block during zygote mitosis or other sensitive periods.

In summary, our results revealed an unexpected zygote mitotic checkpoint, which is extremely sensitive to the CHEK1 kinase activity. The fine-tuning of the DNA damage checkpoint permits the arrested one-cell embryos to overcome the first mitotic block and develop into healthy animals. These findings have important implications in assisted human reproduction and genetic counseling in women with zygotic division failure.

\section{FOOTNOTES}

This work was supported by the National Key R\&D Program of China (Grant 2019YFA0110001, 2017YFA0102802); the National Natural Science Foundation of China (Grant Nos. 31970819, 91740115, 31771108 to JN; Grant No. 32000610 to JG, 82001516 to BC, and 82071724 to ZZ). Beijing Hospitals Authority Youth Programme QML20201401 to LL. JG is supported by the TsinghuaPeking Center for Life Sciences postdoctoral fellowship. Tsinghua University SRT grant 2111S0057 and 2111T0626 to QL and JN. The authors declare no competing interests. All animal experiments were conducted following the Guide for the Care and Use of Animals for Research Purposes. The protocol for mouse embryo isolation was approved by the Institutional Animal Care and Use Committee and Internal Review Board of Tsinghua University.

The human sample collection and analysis was approved by the Ethics Committee for Clinical Medical Research of the First Affiliated Hospital of Anhui Medical University and carried out with informed consent.

The participant has consented to the submission of the study to the journal.

All data generated or analyzed during this study are included in this published article (and its supplementary information files).

All software and codes used this study are included in supplementary information files.

BC, JG, TW, QL, ZZ, LL, YC, and JN conceived the study and designed the experiments. JG, TW, QL, JM performed mouse embryo and oocytes collection and microinjection, phenotype analysis, embryo transfer and transcriptome profiling; BC and FD performed clinical sample collection and data analysis, $\mathrm{HL}$ instructed protein kinase activity study, JG, BC, TW, ZZ, LL, YC and JN wrote the manuscript. All authors read and approved the final version of the manuscript.

Beili Chen ${ }^{1,5}$, Jianying Guo ${ }^{2,6}$, Ting Wang ${ }^{2,6}$, Qianhui Lee ${ }^{3}$, Jia Ming ${ }^{2}$, Fangfang Ding ${ }^{1,5}$, Haitao $\mathrm{Li}^{2,6}$, Zhiguo Zhang ${ }^{1,5 \bowtie}$, Lin $\mathrm{Li}^{4 \bowtie}$, Yunxia Cao ${ }^{1,5 \bowtie}$, Jie $\mathrm{Na}^{2 \bowtie}$

${ }^{1}$ Department of Obstetrics and Gynecology, Reproductive Medicine Center, the First Affiliated Hospital of Anhui Medical University, Hefei 230032, China
${ }^{2}$ School of Medicine, Tsinghua University, Beijing 100084, China

${ }^{3}$ Department of Chemistry, Tsinghua University, Beijing 100084 , China

${ }^{4}$ Central Laboratory, Beijing Obstetrics and Gynecology Hospital, Capital Medical University, Beijing 100026, China

${ }^{5} \mathrm{NHC}$ Key Laboratory of study on abnormal gametes and reproductive tract, Anhui Medical University, Hefei 230032, China

${ }^{6}$ Tsinghua-Peking Center for Life Sciences, Beijing 100084, China $\triangle$ Correspondence: zzg_100@163.com (Z. Zhang), linlithu@ccmu. edu.cn (L. Li), caoyunxia6@126.com (Y. Cao), jie.na@tsinghua. edu.cn (J. Na)

Accepted March 23, 2021

\section{OPEN ACCESS}

This article is licensed under a Creative Commons Attribution 4.0 International License, which permits use, sharing, adaptation, distribution and reproduction in any medium or format, as long as you give appropriate credit to the original author(s) and the source, provide a link to the Creative Commons licence, and indicate if changes were made. The images or other third party material in this article are included in the article's Creative Commons licence, unless indicated otherwise in a credit line to the material. If material is not included in the article's Creative Commons licence and your intended use is not permitted by statutory regulation or exceeds the permitted use, you will need to obtain permission directly from the copyright holder. To view a copy of this licence, visit http:// creativecommons.org/licenses/by/4.0/.

\section{REFERENCES}

Chen L, Chao SB, Wang ZB, Qi ST, Zhu XL, Yang SW, Yang CR, Zhang QH, Ouyang YC, Hou Y et al (2012) Checkpoint kinase 1 is essential for meiotic cell cycle regulation in mouse oocytes. Cell Cycle 11:1948-1955

Eckersley-Maslin MA, Alda-Catalinas C, Reik W (2018) Dynamics of the epigenetic landscape during the maternal-to-zygotic transition. Nat Rev Mol Cell Biol 19:436-450

Emptage RP, Schoenberger MJ, Ferguson KM, Marmorstein R (2017) Intramolecular autoinhibition of checkpoint kinase 1 is mediated by conserved basic motifs of the C-terminal kinaseassociated 1 domain. Journal of Biological Chemistry 292:19024-19033

Goto H, Kasahara K, Inagaki M (2015) Novel Insights into Chk1 Regulation by Phosphorylation. Cell Structure and Function 40:43-50

Han XZ, Tang JS, Wang JN, Ren F, Zheng JH, Gragg M, Kiser P, Park PSH, Palczewski K, Yao XS et al (2016) Conformational Change of Human Checkpoint Kinase 1 (Chk1) Induced by DNA Damage. Journal of Biological Chemistry 291:1295112959

Reichmann J, Nijmeijer B, Hossain MJ, Eguren M, Schneider I, Politi AZ, Roberti MJ, Hufnagel L, Hiiragi T, Ellenberg J (2018) Dual-spindle formation in zygotes keeps parental 
genomes apart in early mammalian embryos. Science 361:189-193

Reinhardt HC, Yaffe MB (2009) Kinases that control the cell cycle in response to DNA damage: Chk1, Chk2, and MK2. Curr Opin Cell Biol 21:245-255
Sidi S, Sanda T, Kennedy RD, Hagen AT, Jette CA, Hoffmans R, Pascual J, Imamura S, Kishi S, Amatruda JF et al (2008) Chk1 suppresses a caspase-2 apoptotic response to DNA damage that bypasses $\mathrm{p} 53, \mathrm{Bcl}-2$, and caspase-3. Cell 133:864-877

Supplementary Information The online version contains supplementary material available at https://doi.org/10.1007/s13238-02100844-9.

Beili Chen, Jianying Guo, and Ting Wang have equal contribution. 